\title{
Application of Progressive Materials for Rapid Prototyping Technology
}

Ing. Ludmila Novakova-Marcincinova, Ing. Miroslav Janak, PhD.

Faculty of Manufacturing Technologies, Technical University of Kosice, Bayerova 1, 08001 Presov, Slovakia

In paper are presented basic information about common and progressive materials used for realization of products by Rapid Prototyping technology application. The initial state of material can come in either solid, liquid or powder state. In solid state it can come in various forms such as pellets, wire or laminates. The current range materials include paper, nylon, wax, resins, metals and ceramics. Last mentioned materials are used manly in application of Rapid Prototyping technology for rapid manufacturing of products or rapid production of tools Rapid Tooling.

Keywords: Rapid Prototyping, rapid prototyping materials, rapid manufacturing

\section{References}

[1] HOPKINSON, N.; HAQUE, R.; DICKENS, P. Rapid Manufacturing: An Industrial Revolution for the Digital Age. Wiley, Oxford, 2006, 304 p., ISBN 978-0470016138.

[2] CHUA, C. K., LEONG, K. F., LIM, C. S.: Rapid Prototyping: Principles and Applications. World Scientific Publishing, Singapore, 2003, 420 p., ISBN 981-238-117-1.

[3] MARCINCINOVA, L. N., FECOVA, V., MARCINCIN, J. N., JANAK, M., BARNA, J.: Effective utilization of Rapid Prototyping technology. Materials Science Forum, Vol. 713, 2012, p. 61-66, ISSN 0255-5476.

[4] MASSOD, S. H., SONG, W. Q.: Development of new metal/polymer materials for rapid tooling using Fused deposition modelling. Materials \& Design, Vol. 25, No. 7, 2004, p. 587-594, ISSN 0261-3069.

[5] SANDA, S., MANAS, M., MANAS, D., STANEK, M., KNOT, J.: Use of Rapid Prototyping in Injection Moulds Design. Mechanical Technology, Vol. 16, No. 5, 2011, p. 58-63, ISSN 1211-4162.

[6] Gallardo Headlight Washer Cover Flap (http://www.crptechnology.com/sito/en/lamborghini-gallardo-headlightwasher-cover-flap.html).

[7] Rapid Prototyping: SLA (http://www.efunda.com/processes/rapid_prototyping/sla.cfm).

[8] Fused Deposition Modeling - FDM (http://www.custompartnet.com/wu/fused-deposition-modeling).

[9] Laser Engineered Net Shaping (http://www.sandia.gov/mst/technologies/net-shaping.html).

\section{Acknowledgements}

Ministry of Education, Science, Research and Sport of SR supported this work, contract VEGA 1/0032/12, KEGA No. $002 T U K E-4 / 2012$ and ITMS project 26220220125.

(1) Al Agentúra $\begin{aligned} & \text { Ministerstva školstva, vedy, výskumu a športu SR } \\ & \text { pre štrukturálne fondy EÚ }\end{aligned}$

